\title{
Intentions vs. Experiences: Opening the Door to Fundamental Conversations About Diversity, Intersectionality, and Race
}

\author{
José M. Flores ${ }^{1} \cdot$ Zheala Qayyum $^{2}$ (D) \\ Received: 17 March 2020 / Revised: 25 June 2020 / Accepted: 8 September 2020 / Published online: 29 September 2020 \\ (C) Academic Psychiatry 2020
}

This Learner's Voice piece describes several encounters between an attending psychiatrist (ZQ) and a psychiatry resident (JF) as they explored the intricacies related to the dynamic definition of the term diversity. The authors have used the terms "Latinx" and "Hispanic" interchangeably in this piece, but we acknowledge that discussions about racial or ethnic identity are fluid and evolving. We also recognize the problematic labeling of American communities from different parts of South/Central/North America, the Caribbean and other parts of the world. There are important subgroups who may not identify with these terms yet may feel culturally connected to the communities or ethnicities discussed in this piece.

\section{Faculty's Viewpoint}

I (ZQ) was drowning in the chaos of the early afternoon on the adolescent inpatient psychiatry unit. Two social workers were trying to talk to me about the pending discharges while the charge nurse was trying to get my attention about a script that one of the parents wanted added to the discharge medications. In the midst of all this, a first-year psychiatry resident, brimming with enthusiasm and having just admitted a new patient on the unit, came by to check in. The resident presented the case and eagerly expressed that he would like to follow the patient. I made a mental note to remind myself to send the residency training directors a note of gratitude for somehow selecting trainees that were a joy to teach, and this young intern too was all about learning. It made my job easier.

Given his own Ecuadorian background, our resident had expressed a lot of interest in following patients with Latin American heritage. There were a few adolescents with

\footnotetext{
Zheala Qayyum

Zheala.Qayyum@childrens.harvard.edu

Yale University School of Medicine, New Haven, CT, USA

2 Harvard Medical School, Boston, MA, USA
}

significant developmental trauma and multiple psychosocial stressors in the unit, even one from Ecuador that we made sure the intern was able to add to his caseload. This new patient too was quite similar. Since it was the resident's last week on the rotation, somehow, I blurted, "Sure, this would be a good case to see, but I think it would be better to have more diversity in the patients you follow."

\section{Learner's Viewpoint}

I (JF) was drowning in the chaos of that early afternoon on the adolescent inpatient psychiatry unit. It was my first month on the child and adolescent psychiatry wards; to say that I was brimming with enthusiasm is to put it mildly. At that moment in my training, everything was new-every case, every demographic, every treatment, and every diagnosis.

I am an Ecuadorian American physician who immigrated to the USA as a young child. I was born in the city of Loja, but it was in the multicultural city of Elizabeth, NJ, that I became bilingual. I give the example of language and bilingualism because Spanish was one of those ever-evident variables used by others to label people like me. But life and labels were not static, and it was during psychiatry residency that I abruptly realized I had lived most of my life in the USA, not in Ecuador. As many Latinxs tell me, I was becoming aware of the complexities and the implications of my pluricultural identity [1]. I was neither Ecuadorian nor American, but somehow, I was both. This identity limbo was even more challenging in medical and graduate school where few people had been in my shoes. To make things even more confusing, the swift changes in my privilege as a physician upon the start of psychiatry residency only exacerbated the conflicts regarding my identity. Certainly, I was a multicultural psychiatrist in training [2].

That afternoon in the psychiatry wards, I evaluated a female pediatric patient who happened to be from Ecuador. She had a history of sexual trauma and a complicated legal 
situation due to differences in laws between both countries. I was appalled by my lack of familiarity with the patient's social, legal, and cultural history. I felt ashamed about my evident ignorance when the patient discussed her trauma with the nuances she would share with, say, a compatriot. She felt that perhaps I would understand. On the contrary, I felt like a fraud, a familiar face with no substantive formulation to help my patient. It was a moment of truth for a painful realization: My acculturation in American society and my isolation from my own Ecuadorian community during medical training had produced glaring deficits in my cultural understanding about the people who were once my own childhood neighbors.

Another patient arrived that afternoon, a male adolescent who also happened to be Ecuadorian. This new patient was older, from a different region of Ecuador, and had a history of trauma and substance use. I realized that both patients were having quite different experiences as Ecuadorian Americans. Their intersectionality was different. I felt this was the perfect opportunity to grow. I had to start fixing my newly perceived deficits in my definition of diversity and learn to see the nuances of different life experiences beyond shared nationality. Eager to make amendments, I asked my attending if I could follow this new patient. My attending replied, "Sure, this would be a good case to see, but I think it would be better to have more diversity in the patients you follow."

My supervisor's words hurt me. I felt that the diversity of my native Ecuador was somehow "insufficient" to qualify for a "bona fide American diversity." At that moment, I felt that I was "too Ecuadorian" to see another Ecuadorian, despite my lack of familiarity with the experiences of people like myself in the American mental health system.

In other situations, I experienced another side of stereotypical assumptions based on a strange list of variables that others thought they saw in me, which presumably collectively measured my level of "Latinoness." For example, Latinx residents often feel the push to evaluate Latinx patients because of an apparent familiarity despite lack of training with these US populations.

\section{The Conversation}

\section{Learner}

I (JF) approached the senior resident hesitantly, with sadness and with fear of the hierarchical system of medicine. I did not want to use the term culturally insensitive to describe one of my favorite teachers but that is how I felt. I also did not want to "play the race card." Many of my colleagues have verbally or non-verbally displayed their aversion to racially charged discussions. However, I could not pretend I was not affected. Thankfully, my senior resident humanized the situation and encouraged me to confide in my attending - he knew that my supervisor was a good person and that she would try to understand these unwelcome feelings.

\section{Faculty}

I (ZQ) heard from the chief resident that the intern had expressed feeling disturbed by this interaction and found it racially and culturally insensitive ("Dr. Q doesn't want me to see Hispanic patients?!'). I felt my heart sink. I, who am very brown, could not fathom how I could be perceived as racially insensitive. But even more, I had offended my trainee, and that was devastating! I have never found myself in this situation before, let alone know how to have a conversation about this. I decided to invite him for a discussion and was somewhat relieved and apprehensive when he came by.

\section{Learner}

The conversation with my attending was difficult. I (JF) did not have the words to express my feelings. Like all trainees, I wanted to perform well in her rotation, I did not want to be a "problem child" in my program, nor did I want to engage in insinuations of misperceived racial transgressions. However, remaining silent made me feel resentful, angry, confused, and, most importantly, complicit. After the first awkward moments, we found ourselves conversing about both of our upbringings. I shared how my experiences had shaped my perceptions of racial insensitivities, whether these came from well-intentioned people or oblivious colleagues. I also told her about my limited experience working with Hispanic/ Latinx patients during medical school. She seemed genuinely surprised.

She told me a little about her experience as a minority and as a woman in academia. I could appreciate her perspective and narrative as I sat there reflecting on mine. It felt safe to share what I understood from her lived experiences. I told her about the pain her words had caused. She heard me. She apologized and told me what she had meant. There was a moment where I realized what diversity meant to me and how much more there is to know about our patients, even if we share similar backgrounds and cultures. She acknowledged how little she knew about this, and I came to realize I did not know much either, certainly I knew less than I thought I did. However, I do remember that at the end we sat there, both humbled, but in mutual respect and admiration of each other. Each a little more grateful for having had this conversation, both a little more enlightened and better understood.

\section{Faculty}

After a brief attempt at slowly stepping around the matter at hand, I (ZQ) mustered the courage to broach the subject. I discovered that my use of the word diversity was understood 
differently than I intended. I was hoping to have him experience greater diagnostic variety, to challenge him to form rapport with patients he did not feel initially drawn to or challenge him to diversify his case load from patients he found it easy to relate with. I felt an urgency to expose him to the multitude of presentations that makes working with adolescents so special. Somehow, I was hoping he would see why I was passionate about all the work we do in child and adolescent psychiatry. In hindsight, the word really did not represent what I wanted to say, or did some aspect of internalized racism just make its way out? There is so much diversity to be appreciated even within patients who may appear similar. Diversity can mean so many things in so many different ways. It took me a moment to realize it. Most importantly, my own minority status does not make me immune to bias or more knowledgeable about the complexities of race, ethnicity, or culture.

\section{Ending Comments}

\section{Faculty}

I (ZQ) did not know the person before me very well and hence found myself having gone down the slippery slope of assumptions. I assumed he would understand what I had meant to say. I assumed I knew about his experience working with the kids on the unit on the basis of my work there for almost 5 years. I had, in the chaos of the circumstances, failed to appreciate the history of cultural, ethnic, racial, or life experiences behind the person who had stood before me.

However, not all my assumptions were incorrect. I had hoped that this conversation would help clear the air and also strengthen the supervisory relationship I had worked very hard to build. I assumed he would hope for the same. That proved to be true. I assumed that if we were respectful and curious about each other's intentions and experience, we would both learn something. That proved to be correct. At the end of the day, what we each carry with us and bring into our work and daily interactions is human, it is our reality, and we can only grow if we open our hearts and just listen.

\section{Learner}

I (JF) walked into my attending's office knowing that we would discuss a very uncomfortable interaction. I prepared myself mentally for the worst as previous experiences with other attendings have been invalidating during cultural dilemmas. Instead, my supervisor's curiosity was uplifting, and her willingness to be vulnerable was inspirational. Ultimately, I realized that when cultural conflicts occur, much more than a trainee's clinical experience is at stake. To invalidate or minimize cultural impasses in the name of a learner's education is unjustifiable precisely because so much more than our education is at stake. As I write this piece, I am filled with gratitude for my attending's leadership as we both grieved and found comfort in our own vulnerabilities. I continue to be excited to see other Ecuadorian American patients and have had the privilege of seeing many more. As my psychiatry training continues, the meaning of diversity in my mind continues to become problematized, and acknowledging its complexity feels just about right, especially if our dream is to truly stand in solidarity with our wonderfully diverse patients, students and colleagues, and with our communities in general.

Acknowledgments We would like to thank Dr. Victor Avila-Quintero, at the Yale Child Study Center, for his support reviewing our work.

\section{Compliance with Ethical Standards}

Disclosures Dr. Flores participates in the Research Training Program in Neuropsychiatric Disorders at Yale Child Study Center and thus receives financial support for his research from NIH through training grant T32MH018268.

\section{References}

1. Goldner-Vukov M. A psychiatrist in cultural transition: personal and professional dilemmas. Transcult Psychiatry. 2004;41(3):386-405.

2. De Figueiredo JM. The making of a multicultural psychiatrist. Transcult Psychiatry. 2011;48(1-2):160-76.

Publisher's Note Springer Nature remains neutral with regard to jurisdictional claims in published maps and institutional affiliations. 